\title{
CALIDAD DE LA EDUCACIÓN Y EL ORIENTADOR EDUCACIONAL
}

\author{
Beatriz Barrero FernándeZ ${ }^{1}$ \\ Jesús Domingo Segovia ${ }^{2}$ \\ JuAn de Dios Fernández GÁlveZ ${ }^{3}$
}

\begin{abstract}
RESUMEN: El artículo aborda el tema del impacto del asesoramiento y el liderazgo intermedio en la mejora educativa de un contexto escolar. Se focaliza en el desempeño profesional de los servicios de apoyo escolar desde su posición estratégica como agentes de mejora y colegas críticos y desde modelos de conexión y colaborativos. Presenta un estudio de caso en una zona educativa que va mejorando significativamente. Se analiza el desempeño profesional de un líder intermedio a partir de la opinión de los interlocutores con los que trabaja. El estudio se desarrolló a lo largo de cuatro cursos académicos. Utiliza grupos de discusión y entrevistas en profundidad. Concluye que la asesoría colaborativa es posible y es valorada positivamente; y que el orientador tiene un rol relevante como líder intermedio.
\end{abstract}

Palabras-clave: Calidad de la educación. Servicio de orientación. Liderazgo. Profesión docente. Interacción.

\section{QUALITY OF EDUCATION AND THE EDUCATIONAL MENTOR}

\begin{abstract}
The article deals with the impact of counselling and middle leadership on educational improvement in a school context. It focuses on the professional performance of school support services from their strategic position as improvement agents and critical colleagues, from connection and collaborative models. It presents a case study in an educational area that is improving significantly. The professional performance of a middle leader is analysed based on the opinion of the interlocutors with whom he or she works. The study was developed over four academic years. It uses discussion groups and in-depth interviews. It concludes that collaborative counselling is possible and positively valued; as well as that the mentor has a relevant role as an intermediate leader.
\end{abstract}

Keywords: Quality of education. School counsellor. Guidance service. Leadership. Teaching profession. Interaction.

Este trabajo forma parte de una sub-línea de trabajo sobre Liderazgo Intermedio, del Proyecto de I + D + i Identidad de la Dirección Escolar: Liderazgo, Formación y Profesionalización [Referencia EDU2016-78191-P].

1.Universidad de Granada - Facultad de Ciencias de la Educación - Granada, España. E-mail: beatrizbarrero@ugr.es

2.Universidad de Granada - Facultad de Ciencias de la Educación - Granada, España. E-mail: jdomingo@ugr.es

3.EOE (Atarfe) Granada. Universidad de Granada - Facultad de Ciencias de la Educación - Granada, España. E-mail: juandefg@gmail.com Editor de sección: Xavier Rambla 


\section{QUALIDADE DA EDUCAÇÃO E O ORIENTADOR EDUCACIONAL}

RESUMO: O artigo trata do impacto da assessoria e da liderança intermediária na melhoria educacional em contexto escolar. Concentra-se no desempenho profissional dos serviços de apoio escolar a partir de sua posição estratégica como agentes de melhoria e colegas críticos, a partir de modelos de conexão e colaboração. Apresenta um estudo de caso em uma área educacional que está melhorando significativamente. O desempenho profissional de um líder intermediário é analisado com base na opinião dos interlocutores com quem ele trabalha. O estudo foi desenvolvido ao longo de quatro anos acadêmicos. Utiliza grupos de discussão e entrevistas detalhadas. Conclui que o aconselhamento colaborativo é possível e é valorizado positivamente; o conselheiro tem um papel relevante como líder intermediário.

Palavras-chave: Qualidade da educação. Serviço de orientação. Liderança. Profissão docente. Inter-relação.

\section{Presentación y Justificación del Problema de Investigación}

$\mathrm{D}$ esde hace más de treinta años, en España, existen unos equipos de orientación zonales con diferentes denominaciones según la Comunidad Autónoma. Normalmente, Equipos Psicopedagógicos de Sector. En Andalucía, Equipos de Orientación Educativa (EOE). Estos equipos de apoyo externo a las escuelas están compuestos por pedagogos, psicólogos o psicopedagogos (bajo el apelativo de "orientadores") que trabajan con un conjunto de colegios de una misma zona educativa. Ello supone una presencia física regular pero intermitente en los mismos.

Hoy en día están suficientemente asentados y consolidados. Se consideran un servicio necesario para el desarrollo de la tarea educativa de calidad, en un momento de transición entre la tradición y la transformación del desempeño profesional (YAVUZ; DAHIR; GUMUSELI, 2017). Desempeñando un conjunto amplio -saturado incluso- de funciones (BISQUERRA, 2008), que tienen lugar en contextos vivos, en escenarios diversos, sujetos en no pocas ocasiones a situaciones urgentes y controvertidas que dificultan la racionalidad y cohesión de su trabajo.

Su rol y funciones están muy bien definidos, pero en la práctica profesional no existe suficiente consenso grupal sobre qué es lo imprescindible de dicha labor y cómo tiene que llevarse a efecto. Cuestión especialmente compleja cuando se trata del trabajo con el resto de profesorado de su zona de influencia, con norte a que rentabilice su trabajo al tiempo que sea más pertinente para generar una buena escuela para todos.

Colabore con los docentes para mejorar el desarrollo del alumnado de manera integral, trabajando en estrecho vínculo con el equipo directivo y promoviendo la innovación en la práctica diaria (MARTÍNEZ; KRISCHESKY; GARCÍA, 2010, p. 110).

Un conjunto de evidencias viene a destacar su labor como colegas críticos que acompañan al profesorado a que redirección en sus esfuerzos hacia la optimización de los procesos de aprendizaje de los estudiantes. Múltiples estudios (SINK, 2009; JANSON; STONE; CLARK, 2009; AMBER; MARTOS, 2017) e informes profesionales (ASCA, 2014; COPOE, 2009; NCTSC, 2009) señalan que pueden contribuir a promover la innovación en la práctica diaria de los centros, a poco que se sientan agentes educativos comprometidos con el progreso del centro más allá de acciones puntuales y en los márgenes del sistema. Pese a ello, algunos estudios resaltan por su controversia 
el asesoramiento educativo como la función más necesaria pero la menos común y demandada (BOZA; TOSCANO; SALAS, 2007).

En la actualidad en España estos servicios de apoyo van recalando en nuevos posicionamientos de actuación profesional colaborativa y comprometida en un proyecto educativo comunitario que permita a todos los agentes educativos ser partícipes de la mejora de los procesos de enseñanza-aprendizaje, encaminados hacia el éxito para todos y entre todos (DOMINGO; FERNÁNDEZ-GÁLVEZ; BARRERO, 2016). Escenario en el que vienen emergiendo posicionamientos que revitalizan su función como líderes intermedios, bisagras del sistema (HARGREAVES; SHIRLEY, 2019) y agentes corresponsables de mejora, desde una perspectiva inclusiva o de justicia social (RYAN, 2017). Como defiende Ainscow (2015), se incrementa el capital social en las escuelas si sus líderes colaboran para apoyar y desarrollar a sus maestros y entre ellos para comprender y emprender juntos mejoras e innovación. Ello parece un nuevo escenario de acción para estos profesionales, que por su posición estratégica e intermedia se encuentran bien ubicados para incidir indirecta y colaborativamente en el ámbito de la política educativa de la escuela, su dimensión dinámica, funcional y cultural y las prácticas de aprendizaje en el aula.

Luego, va consolidándose la perspectiva de que la función actual clave de estos equipos es el asesoramiento psicopedagógico (BISQUERRA, 2008; LAGO; ONRUBIA, 2011; MARTÍN; ONRUBIA, 2011). Como ratifican algunas de estas investigaciones, el orientador como agente ligado a procesos de cambio (MARTÍNEZ; KRISCHESKY; GARCÍA, 2010), líder intermedio y compañero de viaje, se va constituyendo en facilitador y apoyo clave para la innovación y la formación que desemboca en procesos de mejora educativa.

Existen diversidad de formas de entender la labor asesora, pero no cualquier asesoramiento es válido para abordar las funciones tradicionales (ahora bien priorizadas y redimensionadas), con capacidad de implicar a más profesionales en un propósito común. Como se viene describiendo en otro momento, requiere desde una nueva identidad profesional más próxima y un marco de actuación profesional desde un modelo procesual y colaborativoconstructivista, en un rol de "colega crítico" - compañero profesional en el viaje a la mejora- (GURR: HUERTA, 2013; DE LOS MILAGROS; ECHEITA, 2018; DOMINGO, 2009, 2010; LAGO; ONRUBIA, 2011; SWAFFIELD, 2004), para ir conformando un equipo de liderazgo, o grupo motor, por la mejora y la justicia (JANSON; STONE; CLARK, 2009; SINK, 2009; WALKER, 2006).

Una labor profesional específica (en su contexto, funciones y tareas tradicionales) pero que prioriza asumir también labores de liderazgo intermedio con una función cultural y transformadora (RINCÓN, 2019), identificando, optimizando y poniendo en valor las oportunidades estratégicas del contexto, las evidencias, los retos... para cultivar la motivación intrínseca de los colegas con los que trabaja. No obstante, no toda posible colaboración promueve la mejora escolar, se producen disrupciones y dobles mensajes, que desorientan las posibles acciones emprendidas por el propio centro educativo. Por ello, se apuesta a favor de la articulación, la conexión y el apoyo en temas curriculares, cuidando la emoción y la confianza entre ellos y en su labor de cara a la inclusión y el éxito de todos, como motores de cambio y sostenedores de procesos de mejora.

Ello requiere de estos profesionales una reconversión identitaria (DOMINGO; FERNÁNDEZ-GÁLVEZ; BARRERO, 2014; JOHNSON, 2000; STONE; DAHIR, 2015), desde posicionamientos más tradicionales como orientadores técnicos y externos, expertos en diagnóstico e intervención, hacia otros modelos más proactivos y cercanos de "colega crítico", que priorizan sus funciones de acompañamiento y de liderazgo intermedio, para la mejora (de la escuela, del profesorado y de los resultados educativos). Lo que no queda exento de controversias, pudiendo ser percibidas sus acciones, como injerencias en el mundo particular del profesorado (el aula) o entrar en fricción con el propio desarrollo del liderazgo del director.

Como señala Dubar (2002) la reconversión de la identidad profesional supone complejos procesos y crisis identitarias, en las que influyen tanto intereses como prácticas profesionales, resultados y expectativas en interrelación dialéctica. Cuestión central de la que se ocupa este trabajo. De esta manera resulta relevante indagar sobre la identidad 
profesional atribuida y el modelo de actuación profesional de un orientador; así como conocer cómo valora el impacto de estas prácticas profesionales el profesorado, los directivos y la inspección educativa con la que trabaja un orientador que actúa desde esta nueva identidad profesional.

\section{Método}

Se seleccionó de manera intencionada a un orientador de zona. Destacado por compañeros y docentes como una persona particular por su forma de trabajo, como líder intermedio que ejercía una asesoría constructivista y colaborativa con las escuelas y el profesorado. El contexto se caracteriza por ser especialmente retante, escuelas catalogadas por la administración como "de actuación preferente".

El estudio tuvo lugar a lo largo de cuatro cursos académicos, en el que se recogen evidencias de cómo ha sido el proceso de acompañamiento. Al final del ciclo - tras un período de seis cursos en los que el orientador había actuado en estas escuelas- se consideró que era pertinente completar en el estudio de caso el impacto profesional a partir de cómo se percibía y qué valor asignaban los centros al desempeño profesional en esta perspectiva de asesoramiento colaborativo y constructivista.

El impacto percibido por los docentes se midió desde diferentes miradas (las escuelas, los profesionales y directivos de las mismas), en torno a cómo valoran el servicio o qué opinión se tiene de éste y qué demandan o echan en falta del mismo. Se complementó con un análisis de resultados escolares y la perspectiva de los docentes y la inspección educativa.

Se realizaron cuatro grupos de discusión -conformados por la dirección y el equipo técnico de coordinación pedagógica- y una entrevista al inspector de zona. Con ello se produce una triangulación de informantes y perspectivas de análisis. De un lado, internas de cada centro (grupos de discusión) y transversales a los mismos; junto a otras externas, por datos oficiales y, principalmente, por la voz de un profesional externo y experto en la temática, responsable de garantizar la calidad del proceso de asesoría y los resultados en mejora de aprendizaje.

La finalidad de los encuentros era conocer cuál era la visión general de este modelo de actuación del orientador y qué valoración les merecía; en base a las funciones que tenía asignadas y las verdaderamente ejecutadas. Cuál era su valoración y qué imagen, identidad o perfil percibían del orientador estudiado. Finalmente, qué impacto creen que ha tenido su acción profesional en el centro y en los resultados del mismo.

La entrevista en profundidad con el inspector pretendía triangular esta percepción, con más conocimiento del sistema, la normativa y las funciones profesionales que se deben ejercer y los resultados escolares que se han de garantizar.

Para mantener el anonimato, se ha omitido el nombre de los colegios informantes, siendo denominados como A, B, C o D, -según el centro- además de las siguientes claves: director o directora (Dir), jefatura de estudios (JE), profesorado responsable de programas o ciclos (Pro) y la Inspección Educativa de Zona (IE). Para mantener la confidencialidad se pondrá la palabra "Pueblo" o "Localidad" cuando se refieran al nombre del municipio.

Toda la información fue sometida a un análisis temático, con el apoyo de las herramientas de gestión y análisis de información del programa Nvivo 10. Siguiendo la siguiente cascada de profundización reflexiva:

- Desarrollo y análisis de bloques temáticos de los grupos de discusión;

- Análisis temático emergente transversal, a partir de los propios relatos, para obtener las dimensiones clave para los informantes;

- Análisis de resultados educativos en las cuatro escuelas;

- Entrevista en profundidad al inspector de la zona como contraste y validación de la información obtenida desde la mirada de las escuelas. 
Una vez concluido todo este proceso se procedió a la devolución de los resultados obtenidos, los análisis realizados y las conclusiones obtenidas a los informantes para obtener su validación y acuerdo. Del mismo modo se ha realizado una validación dialéctica de contextualización y precisión técnica con el orientador objeto de estudio. Una vez implementado este procedimiento los resultados y análisis se elevaron a definitivos.

En todo momento se respetaron los acuerdos adoptados al inicio de la investigación con los informantes y los planteamientos éticos propios de una investigación de este tipo.

\section{Resultados}

Los resultados se presentan en base a dos bloques: valoración del trabajo del orientador estudiado y demandas hacia estos profesionales que desempeñan una función de bisagra del sistema. Para contextualizar y contratar estos se presenta una evolución de los resultados educativos en las escuelas informantes y se contrasta todo con la perspectiva del supervisor escolar de zona.

\section{La Opinión de las Escuelas}

A modo de síntesis para no perderse en los diferentes árboles que componen este bosque de resultados, se muestra una integración de ideas-clave (Tabla 1), que posteriormente serán desarrolladas en los apartados siguientes.

Tabla 1. Síntesis de ideas de los Grupos de Discusión.

\section{Qué valoran}

Qué demandan

Su peculiar forma de trabajo (lo poco común que es)

Accesibilidad, presencia, participación e implicación continua

Dinamización de propuestas de mejora en base a análisis de evidencias (realidad y resultados) y conocimiento pedagógico

Capacidad de coordinación entre el profesorado (mediación, nexo, apoyo y acompañamiento)

Su trabajo coordinado con otros centros y familias (visión sistémica y de globalidad)

Su preocupación por la comunidad en general

Su labor en la generación, interconexión y articulación de programas de zona y escuelas

Su actuación repercute en un aprendizaje profundo de todos y entre todos (efecto salero)

El poco tiempo que dedica a cuestiones burocráticas, optimizando tiempos de apoyo, acción y formación
Nueva forma de llevar la orientación (colega crítico, colaboración técnica, participación activa en debate y argumentación, promover y dinamizar reflexión curricular/innovación en torno a evidencias $y$ programas...)

Generalizar su forma de trabajo al resto de orientadores

Más presencia física de los orientadores en el centro y en las aulas (en los debates, en la vida y dinámicas del centro, en la toma de decisiones, en el trabajo con otros...)

Tener más programas y directrices técnicas de trabajo específico (valoran la autonomía que estimula, pero demandan más formación, tiempo y técnicas)

Más tiempo para poder realizar una toma de decisiones más reposada

Más diagnósticos e intervenciones específicas (para poder responder a la demanda administrativa)

Nota: Las temáticas resaltadas en cursiva son las más relevantes y objeto de consenso en el conjunto de la información aportada.

Fuente: Elaboración propia. 


\section{Qué Valoran del Trabajo del Orientador Estudiado}

Los resultados denotan que es preciso establecer una premisa de partida. Las percepciones y valoraciones de la actuación profesional del orientador objeto de estudio se refieren a él, a sus características y maneras de ser y actuar, pues desconocen que ello responde a un modelo de trabajo (desde una perspectiva de asesoría colaborativa y constructivista), de la que no poseen referencias previas y desconocen conceptualmente. Valoran e intuyen su potencialidad y virtualidades, pues han participado de los procesos y poseen evidencias de impacto en su profesionalidad, centros educativos e incluso en resultados. Establecido este escenario, los grupos de discusión ponen en valor un conjunto de rasgos, indicadores y temáticas emergentes de los propios discursos, que pasan a ser descritos a continuación.

El primer grupo de discusión A, valoran muy especialmente la accesibilidad que tienen hacia el orientador en todos los temas que afectan a los procesos de enseñanza-aprendizaje y en su desempeño docente. Junto con la accesibilidad, "se implica en todo, aunque no tenga por qué, según las funciones tradicionalmente desarrolladas por estos equipos” (Dir-A). Valoran su actuación profesional pues afirman que su intervención ha cambiado la dinámica de trabajo del centro (innovación educativa y cambio curricular). Destacan la capacidad de dinamización de propuestas promovidas por él, especialmente en temas de inclusión y metodologías y dinámicas que enganchen al alumnado como el trabajo por tareas y proyectos (ABP), sin olvidar su rol de dinamización y enlace entre las escuelas de la localidad.

En el segundo grupo (B), ponen en valor lo poco común que es por su forma de trabajo, su presencia y participación activa. Parece que la tradición vivida con otros profesionales era percibida muy diferentemente (experta y en los márgenes). Destacan la capacidad de nexo y coordinación a nivel de localidad y escuelas. Resaltan su labor en la propuesta de unirse todos los centros educativos de la localidad en torno a algunas de las claves que han propiciado avances posteriores, caminando en una misma línea de trabajo: una escuela inclusiva y para el desarrollo de competencias básicas, el éxito para todos y entre todos, la educación en valores y la acción tutorial. En ello enfatizan su rol de compañero de viaje, dinamizador y mediador, con gran capacidad para impulsar nuevas propuestas y darle coherencia a las mismas desde las necesidades de cada escuela.

Los informantes de la escuela $C$, por su parte, señalan que su forma de llevar a cabo la orientación (desde una perspectiva de asesoramiento curricular colaborativo) es muy positiva, pues rompe completamente con los modelos que hasta el momento habían conocido. Un aspecto llamativo, en este sentido, es que a esta manera de trabajar (colaboración técnica con ellos en lo que "de verdad importa" a maestros y escuelas) le otorgan logros o mejoras palpables en su labor docente, consiguiendo también la clarificación y dotación de sentido de los programas y documentos de centro.

Destacan su capacidad de trabajo, dedicación e implicación; lo sienten comprometido con la mejora emprendida en la escuela y parte de la misma. Valoran este compromiso, su cercanía y su disposición a trabajar con ellos codo con codo. Aprecian su capacidad para promover nuevas propuestas y líneas de acción, y la persistencia que le caracteriza cuando cree que algo es bueno para ellos. Esta particular manera de trabajar junto con el resto de profesores, escuelas de la localidad y con las familias ha permitido que les haya calado la visión de globalidad y de proyecto compartido. Son conscientes de que esta actuación profesional cercana y respetuosa, pero firme y clara, va provocando que todos los colegios trabajen bajo una misma línea, y que en los equipos docentes vayan asumiendo este proyecto colectivo de educación.

Por último, el grupo de discusión de la escuela $D$. valoran su forma de trabajo, destacando la presencia e implicación continua y constante en las propuestas de centro, tanto en la escuela como en la localidad en general. Son conscientes de muchas de las potencialidades que él ha activado en ellos. Afirman que no crea dependencia su forma de actuar, sino que ésta tiene un trasfondo formativo hacia la autonomía del 
profesorado. Al igual que los anteriores centros, resaltan la habilidad para trabajar de forma coordinada con todos los centros de la localidad. Finalmente, conscientes de los requerimientos administrativos, consideran que dedica poco tiempo a las cuestiones burocráticas. Ellos mismos comentan que es un tiempo que invierte a otros asuntos: ver a niños, atender a familias, asesorar al profesorado, etc.

En una mirada transversal se muestran las claves de actuación profesional más destacables en el caso objeto de estudio. Confían plenamente en su competencia técnica en el ámbito de la orientación y la acción tutorial, así como en las actuaciones y respuestas educativas a y en la diversidad que promueve. Eso queda claro, pero lo que verdaderamente destacan es que "se trata de un orientador poco común, que se implica realmente en los programas de centro y en nuestras dinámicas curriculares y organizativas" (Dir-B), lejos de otros desempeños profesionales que "van diagnosticando y recetando sin tiempo de nada más" (JE-C). Una clave,

[...] dedica muy poco tiempo a la gestión burocrática y técnica para dedicarlo a los niños, familias y profesorado. Le da más importancia al asesoramiento de la educación que a la pormenorización y la burocracia (Dir-D).

Coinciden al poner en valor su "accesibilidad y disponibilidad a tiempo completo" (Dir-A), fruto de "una presencia continua en el centro y cerca de las clases" (Pro-D) y su preocupación por la comunidad. "Se trata de una persona con inquietud que crea también inquietud, y eso nos hace crecer y es positivo" (JE-A). "Me gustaría que lo vieses en el trato que tiene con los gitanos y cómo lo ven ellos. Todo un síntoma de mutua aceptación” (Dir-C).

Destacan también la capacidad de dinamización de propuestas desde las necesidades de la escuela. "Participa en todo lo que se implica el colegio" (Dir-A) y de enlazar las mismas con los nuevos aires de innovación educativa que van calando en otros contextos. "Actúa como bisagra y líder intermedio" (IE). Para lo que pone en marcha su capacidad de coordinación entre el profesorado y la localidad desde un trabajo plenamente integrado. Aunque algunas voces lo tildan de "presionante comprensivo",

[...] se preocupó por unificar criterios en cada escuela y aunó como localidad una respuesta educativa, que ha servido de cohesión a todos los colegios de la zona de actuación (Dir-B).

La clave es que está en todas las escuelas, hace la labor de coordinación. Lo que unos trabajamos puede venir bien para otros y él hace esa labor de coordinación y enlace (Dir-A).

En conjunto, se sienten valorados (como verdaderos protagonistas de sus procesos de cambio) y reconocen sus logros en mejoras palpables; ahora bien, sin renunciar a ello, y sintiéndose parte de un equipo, no dudan en coincidir y destacar en ello el destacado rol y la labor de este particular orientador

[...] nos ha ilusionado, hemos transformado nuestra práctica y ahora somos referentes para la Administración Educativa. Si no, iríamos todos a la deriva como íbamos antes (Pro-C).

\section{Qué Demandan al Equipo de Asesoramiento Psico-pedagógico de Zona}

La segunda temática de los grupos de discusión ha versado sobre demandas y reivindicaciones que atiendan a sus retos y necesidades. Por lo común, la temática se dirige en primer término al servicio de apoyo en general, fundamentándose en la experiencia vivida con este orientador a modo de analizador y contraste. 
Seguidamente, conscientes del ritmo de avance que desarrollan y en qué se sienten comprometidos, pero también conscientes de su realidad, posibilidades y circunstancias, unidas a otras presiones administrativas y de funcionamiento del día a día de la escuela, verbalizan otras demandas más particulares y específicas dirigidas al orientador objeto de estudio.

A nivel global, se reclama presencia física y continua de un orientador en los centros y en las aulas, sin que se tenga que ir a otro colegio (escuelas A, B y D). No basta con llegar de manera puntual ante situaciones concretas, sino que estas requieren de un proceso de seguimiento y revisión continua, que implica permanencia en la escuela a tiempo completo. El colegio D reclama más tiempo al orientador para que este pueda ver a todos los niños.

En este sentido el grupo de discusión B demanda que la Administración Educativa otorgue un agente de apoyo de forma permanente en aquellos contextos de difícil desempeño. Tienen necesidad de que se realicen más diagnósticos y más orientaciones de cómo deben trabajar ante la diversidad del alumnado. Esta necesidad va asociada a la gestión de recursos y a la posibilidad de perder la particularidad de ser centro de actuación educativa preferente, con lo que ello comporta en pérdida de recursos y privilegios. Valoran la necesidad de establecer unas líneas o directrices comunes, pero apoyan que estas sean a demanda del centro y no a la inversa. Creen que la orientación debe de partir de la escuela y no para la escuela.

La principal demanda del colegio $C$ gira en torno a fomentar una nueva forma de llevar a cabo la orientación educativa. Donde se establezcan unas directrices claras y se refuerce con una presencia constante de los orientadores en las escuelas. Petición que focalizan hacia la Administración Educativa que es quién determina las funciones y competencias a los EOE. Junto con el anterior centro demandan más actuación diagnostica entrando en controversia con la siguiente afirmación: "Si hubiera más orientadores como él, quizás la cosa a nivel educativo mejoraría bastante" (Dir-C).

Desde una mirada transversal se concluye como principales peticiones generalizar esta forma de trabajo bajo unas líneas o directrices claras "Se tenían que generalizar estos modelos" (Dir-B). Rechazan las actuaciones tradicionales, expertas, técnicas y, en cierto modo, uniformizadoras. En su lugar, solicitan generalizar esta forma de trabajo y se sienten privilegiados en un sistema que cuenta con las mismas oportunidades que ellos.

Frente a nuestra realidad actual, tengo compañeros y compañeras que están en otros colegios que ni siquiera han oído hablar de competencias y trabajan como se ha hecho siempre (Dir-A).

No tiene sentido que venga una persona de fuera, que lo normal no es que sea más un estorbo que ayuda. La mayoría de los centros están descontentos con los equipos de orientación (Pro-B)

A nivel particular, en cuanto a la actuación del orientador objeto de estudio, dos son las demandas principales. De una parte, que sea consciente de que necesitan más tiempo para ir asimilando las propuestas que van emergiendo. "La gente necesita tiempo de ir asimilando los temas [competencias básicas, respuesta educativa, inclusión...]" (JE-A). Y, de otra, que esta presencia física en los centros y en las aulas, redunde también en más actuación diagnóstica, aunque eso es controvertido "Nos faltaría un poco de más tiempo, pero nos hacen falta informes y diagnósticos y él podría hacerlos, pero claro, él tiene el tiempo que tiene” (Pro-D).

\section{Integración de Resultados. Temáticas Emergentes}

Focos de interés emergentes de los propios informantes que se han analizado y que se han mostrado transversalmente consistentes giran en torno a los siguientes analizadores. 


\section{Visión sobre la Orientación: ¿Dos Modelos o Características Personales?}

A nivel general muestran es una visión parcial de las funciones y potencialidades de la orientación educativa. Influidos por su experiencia profesional anterior a la llegada del orientador objeto de estudio a sus vidas y centros, estos profesionales son vistos como profesionales que desarrollan principalmente una acción directa, puntual y resolutiva sobre los niños.

Conozco orientadores de otros centros que se dedican a diagnosticar y planear programas, que tampoco aportan muchas soluciones (Dir- A).

También se han percibido dos visiones contrapuestas, de un lado, cuando se refieren al estudio de caso, tienen una visión positiva y muy bien valorada, de otro, una mirada más escéptica de la tradicional función de los orientadores.

Se han detectado diferencias entre lo que conocen y creen normativamente y las prácticas que les ha tocado vivir. Parece que los receptores no tienen una idea clara de lo que realmente puede hacer un orientador de zona. Identifican solamente funciones tradicionales como el diagnóstico y la evaluación psicopedagógica. Y, se sorprenden cuando conocen otras posibilidades y funciones marcadas en la ley. De esta manera se puede ubicar y entender que desconocen que hay otra manera de trabajar en orientación más colaborativo y socio-constructivista, achacando estos procedimientos necesariamente más democráticos, cercanos, cooperativos e implicativos a condiciones personales y no como elementos clave del modelo de actuación.

\section{Sobre Funciones Profesionales y Actuación Práctica}

Indagando sobre estas funciones, curiosamente, una función más tradicional pero fundamental como sería el asesoramiento continuo y constante ante los problemas diarios con los niños, dando respuesta a la diversidad, sólo es mencionada indirectamente en sólo uno de los centros/grupos de discusión. El resto no la percibe tan clara. Igual es que como es la que consideran más próxima a la identidad atribuida a la orientación, la dan por asentada y obvia, y sólo resaltan los contrastes con la misma.

El tiempo de burocracia lo dedica a atender familias, niños, a profesores y claustros para guiarnos (Dir- D).

El acompañamiento a las familias es otra de las tareas que detectan que realiza.

Se implica en trabajar con el colegio, los niños, los maestros y las familias del colegio (JE-C).

Atiende a las familias orientación familiar y ayuda a niños. Es un orientador muy comprometido. Actúa con familias de muy bajo nivel (Dir- C).

En cambio, como novedad, detectan y valoran que se realice asesoramientos en la elaboración, aplicación y evaluación del Proyecto Educativo (PE). "En este tiempo no solo hemos trabajado el aspecto de orientación a los niños, ha trabajado en todo lo que es el Proyecto Educativo de la escuela" (Dir- A) y "en la construcción de los proyectos de la localidad" (Dir- C). 
También observan que actúa como un apoyo continuo en el diseño de procedimientos e instrumentos de evaluación, tanto de los aprendizajes como de los procesos de enseñanza.

Una tarea que acometió fue que unificáramos todos los criterios de lo que se hacía en cada escuela (JE-B).

Lo primero que se hizo fue ponernos de acuerdo en cuanto a los criterios de evaluación (Dir- B).

Otra función que se detecta en la práctica es su función de nexo, enlace o interlocutor, con otros servicios educativos y sistemas de apoyo, aunque no relacionan esta función a nivel teórico. Comentan al respecto que "también está disponible cuando lo llamas. Asiste al Equipo Técnico y hace de enlace con el Centro del Profesorado y Servicios Sociales" (JE-C), al tiempo que "tiene buenas redes y apoyos" (Dir- C).

La última de las funciones percibidas, de forma práctica, responde a calificarlo como un facilitador de materiales y recursos para la enseñanza.

Si hay alguna duda no le importa venir el día que sea por la tarde. Y siempre que puede viene y nos informa, aporta material y nos escucha (JE-C).

Como se puede apreciar cuando hablamos de funciones percibidas nos damos cuenta que sí que perciben en su forma de actuar las establecidas en la ley. Pero no queda claro que las identifiquen como funciones concretas del orientador. En muchos casos la expresión era de sorpresa cuando describían su forma de trabajo. En el fondo, siguen presentes los modelos tradicionales de actuación. Consideran una suerte con contar con este tipo de asesoría, pero se sienten presionados por la necesidad de contar con diagnósticos, pues son demandados por la propia administración para la provisión de plazas y escolarización.

\section{Impactos Atribuidos a su Desempeño Profesional}

Como cierre de este apartado se destacan algunos impactos que los diferentes grupos de discusión no han dudado en asignar a que en gran medida ha provocado el orientador en los centros educativos. En particular mencionan que tras cuatro años de trabajo se ha creado una agenda local y se ha elaborado de un calendario para las familias del municipio, donde se trabajan valores y se promueven las competencias básicas mediante temáticas locales. Aunque el verdadero impacto de su trabajo viene del cambio de mentalidad y de trabajo que dio lugar a raíz de su llegada. Él ha sido un gran dinamizador de propuestas y estas han tenido una buena acogida.

Ventajas, creo que son evidentes. Sobre todo el cambio de dinámicas de trabajo. Aunque, al mismo tiempo, puede ser un inconveniente porque requiere que el profesorado trabaje más (Dir- A).

\section{La Perspectiva de la Inspección Educativa de Zona}

Indagamos sobre la visión que tenía el inspector, como responsable de la calidad del servicio, sobre el EOE. Esta visión responde a los propios estamentos legales y a la tradición histórica que precede a estos servicios de apoyo. 
En Primaria los orientadores pertenecen a un equipo externo, pero ya forman parte de las escuelas, de todo lo que es la organización de los centros. Están mucho más implicados en la vida de la institución educativa y juegan un papel importante en ella. Si bien desde funciones más especializadas en todo lo que es evaluación psicopedagógica; dictámenes de escolarización; actúan directamente con los alumnos, con las familias y con el profesorado (IE).

Seguidamente, el inspector de zona destaca algunas dimensiones clave del desempeño profesional del orientador y que terminan por generar condiciones organizativas y dinámicas en las que asentar procesos de mejora, como son su capacidad técnica, la colaboración, la implicación, la coordinación y la dinamización de temas, procesos y tareas.

Él entiende la orientación desde la colaboración, desde el trabajo de cooperación y colaboración con las escuelas. Posee la capacidad para integrar entorno a él un equipo de trabajo y lograr que todo el mundo se implique. Esto es clave para lograr cosas (IE).

Es una persona que se implica muy de lleno en los proyectos que al final no solamente es capaz de crear un equipo de trabajo, sino que se implica de una forma permanente y continuada. Responde a las expectativas de los colegios (IE).

Resalta con especial énfasis que tiene la capacidad de actuar como nexo e interconector entre todas las escuelas de la localidad, y su implicación en proyectos van más allá de lo que normalmente sería su labor profesional.

El proyecto de localidad se da porque ha habido una persona [el orientador] que ha sido capaz de aglutinar y de trabajar de manera conjunta. De crear un equipo de trabajo entorno a todos los centros. Él va más allá de lo que es la propia orientación. [...] Mantiene reuniones periódicas con todos los colegios juntos o por separado, y eso hace que todos los centros estén trabajando en una línea determinada; porque tienen un proyecto educativo con principios generales acordados de manera colectiva (IE).

Interesados por saber si ha habido una repercusión en los centros educativos (impactos o indicios de mejora) con la labor del orientador estudiado, el inspector apunta el gran avance que ha acontecido en los últimos tres años en estas escuelas. No lo achaca directamente al orientador, pero tampoco lo desmiente, se ciñe a los datos y evidencias. Eso sí, dados los comentarios precedentes cabe intuir que le reconoce formar parte activa de ello.

Los resultados han sido en los tres años, excepcionales. [...] Se ha logrado en la localidad que las escuelas que tenían más dificultades y peores resultados, por debajo de la media de la provincia y de la comunidad -en competencia matemática, científica, lingüística-, a día de hoy en los últimos resultados todos estén ya en la media andaluza y en algunos casos un poquito por encima. Tan solo en tres años. [...] Y eso sí que significa que están alcanzado mejores resultados escolares. Ahí están los datos. Las estadísticas de los alumnos que obtiene éxito en las distintas materias y en las distintas evaluaciones (IE). 


\section{Datos en Relación a Éxitos Académicos}

Para terminar el análisis se han recopilado los resultados escolares de los últimos seis cursos en términos de éxito académico de los centros de la zona. Es importante señalar que todas las escuelas estaban catalogadas con un bajo índice socio-económico y cultural, y pese a ello han vivido un avance hacia la mejora de resultados. Estos datos sólo se aportan con objeto de contextualización y sin buscar relaciones causales entre otras posibles variables vinculadas a la función de orientación. Se presentan tantos los porcentajes globales de aprobados como los del alumnado con varias materias suspensas, por ciclos y totales, de las cuatro escuelas de la localidad en su conjunto (Tabla 2).

Tabla 2. Resultados globales de zona educativa, comparativa entre el primer año de intervención y el cuarto.

\begin{tabular}{lcccc}
\hline Indicadores en $\%$ & Ciclo & Evaluación $\mathbf{1}^{\mathbf{0}}$ año & Evaluación $\mathbf{4}^{\mathbf{o}}$ año & Diferencial \\
\hline & 1 er Ciclo & $73,9 \%$ & $89,2 \%$ & $+15,3 \%$ \\
Alumnado que & $2^{\circ}$ Ciclo & $70,9 \%$ & $79,3 \%$ & $+8,4 \%$ \\
aprueba todo & er Ciclo & $60,5 \%$ & $77,9 \%$ & $+17,4 \%$ \\
& Total & $68,4 \%$ & $82,1 \%$ & $+13,7 \%$ \\
\hline \multirow{2}{*}{ Alumnado que } & 1 er Ciclo & $8 \%$ & $1,7 \%$ & $-6,3 \%$ \\
suspende más de & $2^{\circ}$ Ciclo & $14,9 \%$ & $7,2 \%$ & $-7,7 \%$ \\
cuatro materias & 3er Ciclo & $10,4 \%$ & $8,6 \%$ & $-1,8 \%$ \\
& Total & $11,1 \%$ & $5,8 \%$ & $-5,3 \%$ \\
\hline
\end{tabular}

Fuente: Elaboración propia.

Estos resultados muestran que efectivamente ha aumentado casi en un $14 \%$ el número de alumnos que aprueban todo. En el curso de partida la media de alumnado que aprobada era de un $68,4 \%$ frente a un $82,1 \%$ de los datos del último curso. Al mismo tiempo, ha disminuido la tasa de alumnos que terminan con más de cuatro asignaturas suspensas. De un $11,1 \%$ de alumnos con al menos cuatro materias suspendas a un $5,8 \%$ Bajando el porcentaje casi al 50\%. Lo que sin duda es un dato altamente significativo y gratificante que denota que la zona educativa ha avanzado. Lo cual, junto a la intervención de otros factores que no se han controlado en el estudio puede venir por esta presencia activa y asesoría colaborativa (a directivos y profesorado) que ha venido prestando el orientador a estas cuatro escuelas.

\section{Discusión de Resultados, Conclusiones e Implicaciones}

Los resultados de este estudio evidencian la percepción y la valoración que hacen los interlocutores, los agentes profesionales con los que trabaja el orientador objeto de estudio.

Se han detectado diferencias entre lo que conocen y creen normativamente y las prácticas que les ha tocado vivir. Parece que los receptores no tienen una idea clara de lo que realmente puede hacer un orientador de zona. Identifican solamente funciones tradicionales como el diagnóstico y la evaluación psicopedagógica. Y, se sorprenden cuando conocen otras posibilidades y funciones marcadas en la ley. Con ello identifican las bondades en la actuación profesional o la diferente manera de abordar situaciones y funciones tradicionales (como el diagnóstico o la implicación profesional), pero no las ubican en torno a un nuevo modelo de enfocar la orientación, colaborativo y socio-constructivista (DOMINGO; FERNÁNDEZ-GÁLVEZ; BARRERO, 2014, 2016), y lo achacan a características personales del orientador. 
De una parte, parece que sigue presente y demandan la imagen del orientador reactivo, que diagnostica y soluciona problemas. Pero, por otro lado, cuando estos vivencian otros modelos de apoyo, más pedagógicos, manifiestan una gran satisfacción. En el discurso de los interlocutores consultados se observan también controversias. Como ya denunciara Johnson (2000) en este sentido, aún persisten dificultades para definir con precisión el rol del consejero escolar especialmente desde el profesorado (BOZA; TOSCANO; SALAS, 2007), y se observa como miran con esperanza, pero también con prudencia y cierto recelo, otros modelos de desempeño profesional diferentes a los tradicionales, aunque terminen siendo más efectivos y mejor valorados (YAVUZ; DAHIR; GUMUSELI, 2017).

Este orientador es un vivo ejemplo de que, trabajando así, tras un periodo de reajuste y cambio de expectativas del profesorado, los resultados son evidentes. Se valora el cambio de modelo, pero se tienen dudas ante el aparente "abandono de la atención individual a alumnado" (diagnóstico tradicional y adaptaciones curriculares). Y, sobre todo, desde la dirección de las escuelas -más conocedoras de los procedimientos administrativos. Se teme la pérdida de recursos (de atención a la diversidad) por la caída en el número de diagnósticos e informes burocráticos para la administración. El apoyo explícito de la administración a desempeños profesionales más implicados cultural e institucionalmente, dentro de esta perspectiva (BOLÍVAR, 2008), quitaría incertidumbres y optimizaría los muchos esfuerzos poco rentables de muchos otros profesionales.

A lo largo del trabajo se ha podido dibujar una imagen social (identidad atribuida que dijera Dubar [2002]) del perfil del orientador. De los comentarios recogidos destacan rasgos e indicadores que responden a un desempeño profesional cercano, comprometido y guiado por la colaboración, el acompañamiento, la implicación y participación continua, como motores capaces de impulsar propuestas, gestionarlas y donarlas para el centro y la localidad. En definitiva, un modelo de asesoramiento colaborativo-constructivista con vocación curricular y de localidad, con potencial para transformar una zona educativa desde la labor del orientador (DOMINGO; FERNÁNDEZ-GÁLVEZ; BARRERO, 2014, 2016) compatible con lo recomendado por la literatura actual sobre asesoría (DE LOS MILAGROS; ECHEITA, 2018; DOMINGO, 2009; HOPKINS, 2007; SINK, 2009; JANSON; STONE; CLARK, 2009; LAGO; ONRUBIA, 2011).

Ahora bien, es preciso reconocer que, como han verbalizado los informantes, el orientador objeto de estudio es particular y presenta un modelo de desempeño profesional en su zona educativa diferente a lo vivido previamente. Este modelo constructivista, dialógico y colaborativo con una perspectiva de localidad es poco común (desconocido incluso para ellos) y difícilmente generalizable, parece evidente que no es lo común (lo esperado) de estos servicios de apoyo, aunque es ampliamente reconocido y, ahora, demandado. Pese a esta particularidad, el caso se aproxima bastante a las orientaciones de asociaciones profesionales (ASCA, 2014; COPOE, 2009; NCTSC, 2009).

El discurso de sus interlocutores pone de manifiesto también su acción como líder intermedio y como interconector entre escuelas, haciendo red en torno a proyectos comunitarios, es un modelo interesante, pertinente y que se ajusta a las orientaciones de la administración educativa y a la literatura actual sobre el tema. En particular, coincide con algunos lineamientos destacados en la literatura sobre liderazgo intermedio (BOLÍVAR, 2014; DASTMALCHIAN et al., 2016; HARRIS; JONES, 2012; RINCÓN, 2019) en un contexto en el que importa el capital social y profesional (AINSCOW, 2015). Los resultados ponen en evidencia que este orientador trabaja desde esta perspectiva, buscando el desarrollo de una visión compartida, utilizando el acompañamiento, la escucha activa, la disponibilidad y la implicación como escenario en el que pone en valor e implica a los otros líderes intermedios propios o cercanos a la escuela, como líderes de responsabilidad (SINK, 2009). 
A juicio de los interlocutores consultados, el proceso de acompañamiento y asesoría colaborativa por parte de este orientador escolar de zona ha coadyuvado a transformar las escuelas en las que actúa y a dar respuestas educativas con impacto en la mejora de resultados escolares. Señalan claramente que, ahora, con el apoyo y acompañamiento del orientador, avanzan hacia una escuela más inclusiva, un currículum más democrático, evaluaciones más formativas y el inicio de metodologías que facilitan el enganche del alumnado y mejores resultados educativos.

En el estudio de caso también se ha mostrado que las escuelas valoran estas funciones y desempeño profesional e instan a la administración educativa a que ponga en valor las funciones que se centran en el asesoramiento y el cambio. Priorizar las intervenciones globales frente a las individuales, orientadas por la colaboración, así como las de interrelación interprofesional. Lo que coincide con el trabajo de FernándezLarragueta; Fernández-Sierra; Rodrigo (2014).

Sin poder establecer relaciones de causalidad entre la labor profesional objeto de estudio y mejora de resultados educativos, no era el propósito del estudio ni se han controlado variables al respecto, la verdad es que dadas las valoraciones vertidas en este sentido por los interlocutores se consideró relevante tomarlos en consideración para comprender mejor el caso. Mucho más cuando la mejora global de resultados académicos es evidente a medio plazo (unos pocos años). Puede ser que, como defiende Bolívar (2014), el ejercicio del acompañamiento y coliderazgo que ha evidenciado el caso haya podido servir en alguna medida para empoderar al profesorado y al centro como comunidad profesional. El impacto provocado en la zona educativa por esta forma de trabajo a lo largo del tiempo es un ejemplo más, coincidente con las conclusiones de Cano y García-Nieto (2010), que remarca la bondad de la transición del modelo tradicional de orientación remedial y de intervención, hacia otro basado precisamente en la colaboración, la participación, la interrelación profesional y el asesoramiento como "colega crítico". Lo que implica más implicación de los centros educativos y su profesorado en los procesos de mejora, en consecuencia, mayor posibilidad de permanencia y sostenibilidad de los mismos, más allá de la presencia técnica activa del propio asesor.

En definitiva, el caso apunta directrices de que los maestros deben ser diseñadores de potentes situaciones de aprendizaje y que los orientadores pueden ser buenos acompañantes en este proceso. Actuando desde la sombra, sin que su influencia sea directiva, sino facilitadora y abogando por el desarrollo de una identidad profesional híbrida, como colega crítico y líder intermedio (BARRERO; DOMINGO; FERNÁNDEZGÁLVEZ, 2020). Una de las claves del trabajo de los orientadores como asesores gira en torno a hacer que las demandas sean del centro y no para el centro, luego su modelo de desempeño profesional debe virar hacia modelos más horizontales y de interconexión, sin obviar su labor de (co)liderazgo, teniendo la colaboración y el compromiso profesional como señas de identidad (DE LOS MILAGROS; ECHEITA, 2018). La otra clave, también muy substantiva, es que sin unos resultados de mejora educativa (para todos), la promoción de la identidad profesional del asesoramiento escolar es simplemente "humo y espejos" (JOHNSON, 2000). Por tanto, $y$, sin pretensión de generalizar resultados, el caso aporta evidencias de interés que pueden ayudar a nutrir el debate en torno a cómo desempeñar, con impacto positivo en su contexto, la asesoría educativa y el liderazgo pedagógico desde las bisagras del sistema.

\section{Contribuciones de los Autores}

Planteamiento del problema y contextualización; Domingo J. Metodología y análisis; Barrero B. Redacción; Domingo J, Fernández Gálvez J, Barrero B. 


\section{Referencias}

AMBER, D.; MARTOS, M. A. Ámbitos y funciones de los orientadores para la mejora educativa en secundaria en contextos retantes. Una mirada cruzada entre orientadores y directivos. Profesorado - Revista de Currículum y Formación del Profesorado, Granada, v. 21, n. 4, p. 419-437, 2017. https://doi.org/10.30827/profesorado.v21i4.10063

AINSCOW, M. Towards Self-Improving School Systems: Lessons from a City Challenge. London: Routledge, 2015. ASCA [AMERICAN SCHOOL COUNSELOR ASSOCIATION]. Careers/roles: what does a school counselor do? All Psychology Schools, 2014. Disponible en: https://www.allpsychologyschools.com/school-counseling/jobdescription/. Acceso en: 15 dez. 2019.

BARRERO, B.; DOMINGO, J.; FERNÁNDEZ-GÁLVEZ, J. D. Liderazgo intermedio y desarrollo de comunidades de práctica profesional: lecciones emergentes de un estudio de caso. Psicoperspectivas, Valparaíso, v. 19, n. 1, p. 1-13, 2020.

BISQUERRA, R. Orientación psicopedagógica: áreas y funciones. In: SECRETARIA GENERAL DE EDUCACIÓN. Funciones del Departamento de Orientación. Madrid: Instituto Superior de Formación y Recursos en Red para el Profesorado, 2008. p. 11-39. (Conocimiento Educativo.)

BOLIVAR, A. Orientación y educación para la ciudadanía. In: SECRETARIA GENERAL DE EDUCACIÓN. Funciones del Departamento de Orientación. Madrid: Instituto Superior de Formación y Recursos en Red para el Profesorado, 2008. p. 235-266.

BOLÍVAR, A. Construcción de capacidades de la escuela: liderazgo compartido y comunidades profesionales de aprendizaje. Una propuesta de investigación. International Journal of Educational Leadership and Management, Barcelona, v. 2, n. 2, p. 147-175, 2014.

BOZA, Á.; TOSCANO, M. O.; SALAS, M. ¿Qué es lo que hace un orientador? Roles y funciones del orientador en Educación Secundaria. XXI - Revista de Educación, v. 9, p. 111-131, 2007.

CANO, J. G. N. Aproximación a la percepción de los orientadores escolares sobre la colaboración que reciben de la comunidad educativa: retos y propuestas. Revista Complutense de Educación, Madrid, v. 2, n. 11, p. 149-169, 2010.

CLARK, M. A.; CRANDALL, J. School counselor inclusion: a collaborative model to provide academic and socialemotional support in the classroom setting. Journal of Counseling and Development, New York, v. 87, n. 1, p. 6-11, 2009. https://doi.org/10.1002/j.1556-6678.2009.tb00543.x

COPOE [CONFEDERACIÓN DE ORGANIZACIONES DE PSICOPEDAGOGÍA Y ORIENTACIÓN DE ESPAÑA]. Conclusiones y reivindicaciones de los profesionales de la Orientación. I Jornadas de Orientadores, Murcia, 24 abr. 2009. Disponible en: www.copoe.org. Acceso en: 2 nov. 2019

DASTMALCHIAN, A. et al. Developing a measure for "connectorship" as a component of engaged leadership. Leadership \& Organization Development Journal, Bradford, v. 37, n. 3, p. 403-427, 2016. https:/doi.org/10.1108/ LODJ-07-2014-0135

DE LOS MILAGROS, M. L.; ECHEITA, G. Un departamento de orientación singular en un centro educativo que tiene la colaboración como seña de identidad. Profesorado - Revista de Currículum y Formación del Profesorado, Granada, v. 22, n. 2, p. 161-183, 2018. 
DOMINGO, J. Asesoría a la escuela para la mejora de la enseñanza y el aprendizaje. In: UNIVERSIDAD PEDAGÓGICA NACIONAL. Seminario Internacional Itinerante. Desarrollo de la gestión educativa en México: situación y perspectivas. Ciudad de México: UPN, 2009. p. 101-126.

DOMINGO, J. Comprender y redireccionar las prácticas de asesoría. Revista Iberoamericana de Educación, v. 54, p. 65-83, 2010.

DOMINGO, J.; FERNÁNDEZ-GÁLVEZ, J. D.; BARRERO, B. La función de asesoría para la mejora de la enseñanza y el aprendizaje en la práctica profesional de un orientador de zona. Un estudio de orientador. Educatio Siglo XXI, Murcia, v. 32, n. 2, p. 151-172, 2014. https://doi.org/10.6018/j/194131

DOMINGO, J.; FERNÁNDEZ-GÁLVEZ, J. D.; BARRERO, B. El orientador escolar ante el reto de la mejora curricular. Un estudio de caso. Revista Electrónica de Investigación Educativa, Ensenada, v. 18, n. 2, p. 27-39, 2016.

DUBAR, C. La crisis de las identidades. La interpretación de una mutación. Barcelona: Bellaterra, 2002.

FERNÁNDEZ-LARRAGUETA, S.; FERNÁNDEZ-SIERRA, J.; RODRIGO, M. Coordinación interprofesional en los centros educativos: una apuesta para la inclusión. Estudios sobre Educación, v. 27, p. 193-211, 2014.

GURR, D.; HUERTA, M. The role of the critical friend in leadership and school improvement. Procedia-Social and Behavioral Sciences, Red Hook, v. 106, p. 3084-3090, 2013. https://doi.org/10.1016/j.sbspro.2013.12.356

HARGREAVES, A.; SHIRLEY, D. Leading from the middle: its nature, origins and importance. Journal of Professional Capital and Community, Bingley, v. 5, n. 1, p. 92-114, Dec. 2019. https://doi.org/10.1108/JPCC-06-2019-0013

HARRIS, A.; JONES, M. Connecting professional learning: leading effective collaborative enquiry across teaching alliances. Nottingham: National College for School Leadership, 2012.

HOPKINS, D. Every school a great school. Realizing the potential of system leadership. New York: McGraw Hill/ Open University Press, 2007.

JANSON, C.; STONE, C.; CLARK, M. A. Stretching leadership: a distributed perspective for school counselor leaders. Professional School Counseling, Alexandria, v. 13, n. 2, p. 98-106, 2009. https://doi.org/10.1177/2156759X0901300205

JOHNSON, L. S. Promoting professional identity in an era of educational reform. Professional School Counseling, Alexandria, v. 4, n. 1, p. 31-40, 2000.

LAGO, J.R.; ONRUBIA, J. Una estrategia general de asesoramiento para la mejora de la práctica educativa. Profesorado -Revista de Currículum y Formación del Profesorado, Granada, v. 12, n. 1, p. 1-13, 2008.

LAGO, J. R.; ONRUBIA, J. Asesoramiento psicopedagógico para la mejora de la práctica educativa. Barcelona: ICE-Horsori, 2011.

MARTÍN, E.; ONRUBIA, J. (coords.). Orientación educativa. Procesos de innovación y mejora de la enseñanza. Barcelona: Graò, 2011.

MARTÍNEZ, C. A.; KRISCHESKY, G. J.; GARCÍA, A. El orientador escolar como agente interno de cambio. Revista Iberoamericana de Educación, Madrid, v. 54, p. 107-122, 2010.

MONEREO, C.; POZO, J. I. (coords.). La práctica del asesoramiento educativo a examen. Barcelona: Graó, 2005.

NCTSC [NATIONAL CENTER FOR TRANSFORMING SCHOOL COUNSELING]. The new vision for 
school counselors: scope of the work. The Educational Trust, 2009. Disponible en: http://www.edtrust.org/ dc/tsc/vision. Acceso en: 26 nov. 2019

RINCÓN, S. Las redes escolares como entornos de aprendizaje para los líderes educativos. In: WEINSTEIN, J.; MUÑOZ, G. (eds.). Cómo cultivar el liderazgo educativo. Trece Miradas. Santiago de Chile: Ediciones Universidad Diego Portales, 2019. p. 355-388.

RYAN, J. Un liderazgo inclusivo para las escuelas. In: WEINSTEIN, J. (ed.). Liderazgo educativo en la escuela: Nueve miradas. Santiago de Chile: Ediciones Universidad Diego Portales, 2017. p. 177-204.

SINK, C. School counselors as accountability leaders: another call for action. Professional School Counseling, Alexandria, v. 13, n. 29, p. 68-74, 2009. https://doi.org/10.1177/2156759X0901300202

STONE, C.; DAHIR, C. A. The transformed school counselor. Toronto: Nelson Education, 2015.

SWAFFIELD, S. Critical friends: supporting leadership, improving learning. Improving Schools, London, v. 7, n. 3, p. 267-278, 2004. https://doi.org/10.1177/1365480204049340

WALKER, J. Principals and counsellors working for social justice: a complementary leadership team. Guidance and Counseling, Colorado Springs, v. 21, n. 2, p. 114-124, 2006.

YAVUZ, O.; DAHIR, C.; GUMUSELI, A. I. School principal perceptions of the school counsellor's role: traditional or transformed? Journal of Educational Leadership, Policy and Practice, Auckland, v. 32, n. 2, p. 81-97, 2017. https:// doi.org/10.21307/jelpp-2017-0021

\section{Sobre los Autores}

Beatriz Barrero Fernández es Doctora en Pedagogía por la Universidad de Granada. Profesora Ayudante Doctora en el Departamento de Didáctica y Organización Escolar de la Universidad de Granada. Miembro del Proyecto Atlántida. Su línea de investigación actual versa sobre Identidad profesional y Asesoría educativa.

Jesús Domingo Segovia es Doctor en Pedagogía por la Universidad de Granada. Catedrático de Universidad en el Departamento de Didáctica y Organización Escolar de la Universidad de Granada. Editor Adjunto de Profesorado Revista de Currículum y Formación del Profesorado. Miembro de la Red de Investigación sobre Liderazgo y Mejora Educativa (RILME). Miembro del Proyecto Atlántida. Su línea de investigación actual versa sobre Liderazgo desde el medio y asesoría para la mejora de la calidad y la equidad en educación e Investigación (auto)biográfica en educación.

JuAn de Dios Fernández Gálvez es Licenciado en Psicología y Doctor en Pedagogía por la Universidad de Granada. Profesor Asociado en el Departamento de Didáctica y Organización Escolar dela Universidad de Granada. Orientador de Equipo de Orientación Educativa. Director de la Delegación Provincial de Educación de la Junta de Andalucía. Miembro del Proyecto Atlántida. Su línea de investigación actual versa sobre Escuela Inclusiva; Desarrollo profesional docente y desarrollo curricular para el logro de los Objetivos de Desarrollo Sostenible.

Recibido: 23 jan. 2020

Aceptado: 23 mar. 2021 